were defined. Among these proteins, vascular cell adhesion molecule-1 (VCAM1) was found increased in HOA compared to RA and PSA groups.

Conclusions: A specific protein profile for the characterisation of EHOA and NHOA disorders has been established. VAS showed elevated levels in patients with $\mathrm{NHOA}$, whereas ECM1 was increased in patients diagnosed with the erosive form of the disease. As none of them were identify in the other phenotype, they might be phenotype-specific biomarkers. In addition, VCAM1 was found with higher levels in both phenotypes of HOA when compared with RA and PSA and might be used to differentiate hand osteoarthritis from other rheumatic diseases. Acknowledgements: Financial support (IN606A-2016/012) from the Xunta de Galicia and the European Union (European Social Fund - ESF), is gratefully acknowledged.

Disclosure of Interest: None declared

DOI: 10.1136/annrheumdis-2018-eular.4495

\section{FRI0521 COMBINING FRACTAL- AND ENTROPY-BASED BONE TEXTURE ANALYSIS FOR THE PREDICTION OF OSTEOARTHRITIS: DATA FROM THE MULTICENTER OSTEOARTHRITIS STUDY (MOST)}

R. Ljuhar ${ }^{1}$, Z. Bertalan ${ }^{1}$, S. Nehrer ${ }^{2}$, B. Norman ${ }^{3}$, H.-P. Dimai ${ }^{4}$, A. FahrleitnerPammer $^{4}$, D. Ljuhar ${ }^{3} .{ }^{1}$ Research and Development, ImageBiopsy Lab, Vienna; ${ }^{2}$ Center for Regenerative Medicine and Orthopedics, Danube University, Krems, Krems; ${ }^{3}$ Research and Development, Braincon Technologies, Vienna; ${ }^{4}$ Department of Internal Medicine, Division of Endocrinology and Diabetology, Medical University of Graz, Graz, Austria

Background: Osteoarthritis $(O A)$ is one of the leading causes of long-term pain and disabilities associated with musculoskeletal disorders. Effective treatment and disease-progression slowdown depend on early detection and quantification of risk. However, current disease parameters, like joint space width (JSW), have proven to be insufficient for the prediction of $O A$.

Objectives: The purpose of the present study was to investigate if combining fractal- and entropy-based bone texture analyses with joint space width (JSW) and joint space area (JSA) may improve prediction of OA. Conventional posterioranterior (PA) knee radiographs of men and women were obtained from the Multicenter Osteoarthritis Study (MOST) database, which provides valuable information to identify and define modifiable biomechanical, bone and structural, nutritional, and other risk factors for new disease and progression of existing disease.

Methods: Oriented fractal- and entropy based texture algorithms were developed, using state-of-the-art computer hardware and software as well as specific machine-learning algorithms. The selected subchondral area used for textural analyses included 4 regions of interest $(\mathrm{ROI})$ in the proximal tibia and one on each condyle of the distal femur (figure 1). Furthermore, JSW and JSA were assessed using newly developed and fully automated software.

Results: 1092 conventional knee radiographs obtained from one study centre were screened for eligibility. Of these, a total of 574 radiographs ( 230 women, 344 men) met the inclusion criteria, i.e. a Kellgren \& Lawrence (KL) score of 0 at baseline. At month 84, 41 female and 79 male patients had developed $K L>1$, and 189 female and 265 male patients remained at KLO. Area-Under-the-Curve (AUC) for incident OA using JSW/JSA and clinical features was $0.67 \pm 0.08$ for women, and $0.61 \pm 0.1$ for men. In contrast, combining fractal/entropy-based texture, JSW/A and clinical features resulted in significantly improved AUC for women and men $(0.80 \pm 0.07$ for women and $0.69 \pm 0.1$ for men, respectively). To test whether these differences in predicting incident-OA were significant, we performed classifier comparison: $t=3.84 ; p<10-3$ for women, and $t=3.38 ; p<10-3$ for men.

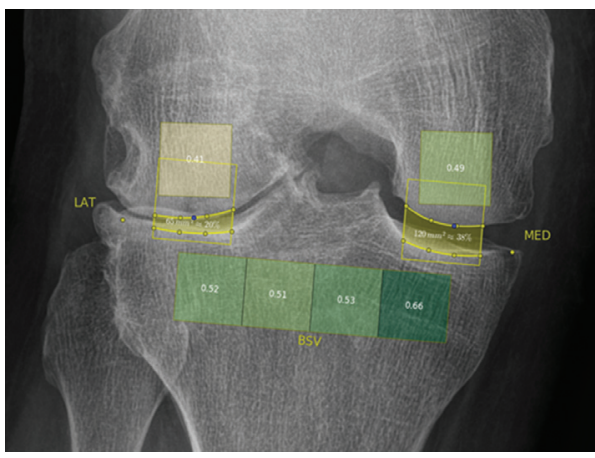

Conclusions: This study provides strong evidence, that a combination of fractaland entropy-based textural analyses of plain subchondral bone radiographs together with JSW/A and clinical features is superior to JSW/A and clinical features alone in predicting incident $\mathrm{OA}$ in men and women.
REFERENCE:

[1] http://most.ucsf.edu/studyoverview.asp

Disclosure of Interest: R. Ljuhar Shareholder of: ImageBiopsy Lab, Z. Bertalan : None declared, S. Nehrer: None declared, B. Norman : None declared, H.-P Dimai : None declared, A. Fahrleitner-Pammer: None declared, D. Ljuhar: None declared

DOI: 10.1136/annrheumdis-2018-eular.1118

\section{FRI0522 VITAMIN D SUPPLEMENTATION IMPROVES DEPRESSION IN KNEE OSTEOARTHRITIS PATIENTS OVER 24 MONTHS}

S. Zheng ${ }^{1}$, L. Tu${ }^{2}$, F. Cicuttini ${ }^{3}$, W. $\operatorname{Han}^{1}$, Z. Zhu ${ }^{1}$, B. Antony ${ }^{1}$, A. Wluka ${ }^{3}$, T. Winzenberg ${ }^{1,4}$, D. Aitken ${ }^{1}$, L. Blizzard ${ }^{1}$, G. Jones ${ }^{1}$, C. Ding ${ }^{1,5} .{ }^{1}$ Menzies Institute for Medical Research, University of Tasmania, Hobart, Australia; ${ }^{2}$ Department of Rheumatology, The third Affiliated Hospital of SUN YAT-SEN University, Guangzhou, China; ${ }^{3}$ Department of Epidemiology and Preventive Medicine, Monash University, Melbourne; ${ }^{4}$ Faculty of Health, University of Tasmania, Hobart, ${ }^{5}$ Clinical Research Centre, Zhujiang Hospital, Southern Medical University, Guangzhou, Australia

Background: Although depression is prevalent in osteoarthritis (OA) patients and the positive association between vitamin $\mathrm{D}$ deficiency and depression has been demonstrated, no study has examined the effect of vitamin D supplementation on depression in OA patients.

Objectives: To determine the effect of vitamin D supplementation and maintain ing sufficient serum vitamin $D$ on depression in patients with knee $O A$ and vitamin $\mathrm{D}$ deficiency.

Methods: Participants with symptomatic knee OA and vitamin D deficiency were enrolled in a randomised, placebo-controlled trial and received 50,000IU vitamin $D_{3}(n=209)$ or placebo $(n=204)$ monthly for 24 months. Serum 25 -hydroxyvitamin D $[25(\mathrm{OH}) \mathrm{D}]$ was measured at baseline, month 3 and 24. Depression was measured using the patient health questionnaire (PHQ-9), and knee symptoms were assessed using Western Ontario and McMaster Universities Arthritis Index (WOMAC) at baseline, month 3, 6, 12 and 24. Multilevel mixed-effect models were used to estimate the association between exposures and outcomes adjusting for potential confounders.

Results: Over 24 months, 340 participants (82.3\% retention rate) completed the study. The prevalence and incidence of depression were $25.4 \%$ and $11.2 \%$, respectively. Depression improved more in the vitamin $\mathrm{D}$ supplementation group ( $\beta$ : $-0.45,95 \% \mathrm{Cl}:-0.84$ to -0.07 ) compared to the placebo group ( $\beta$ : 0.21 $95 \% \mathrm{Cl}:-0.19$ to 0.61$)(\mathrm{p}=0.02)$ and in those participants who maintained vitamin D sufficiency ( $\beta$ : $-0.44,95 \% \mathrm{Cl}:-0.88$ to -0.00 ) compared to those who did not maintain sufficiency $(\beta: 0.40,95 \% \mathrm{Cl}$ : -0.18 to 0.97$)(p=0.02)$ over 24 months.

Abstract FRI0522 - Table 1. Effects of vitamin D supplementation over 24 months on change in PHQ-9

\begin{tabular}{lccc}
\hline & $\begin{array}{c}\text { Mean change, } \\
(95 \% \mathrm{Cl})\end{array}$ & $\begin{array}{c}\text { Between-group difference change, mean } \\
(95 \% \mathrm{Cl})\end{array}$ & $\begin{array}{c}\mathrm{P} \\
\text { value }\end{array}$ \\
\hline $\begin{array}{l}\text { Placebo } \\
\text { Group }\end{array}$ & $0.21(-0.19$ to 0.61$)$ & $-\mathbf{0 . 6 6}(-\mathbf{1 . 2 2}$ to $-\mathbf{0 . 1 1})$ & $\mathbf{0 . 0 2}$ \\
$(\mathrm{n}=204)$ & & \\
$\begin{array}{l}\text { Vitamin } \mathrm{D} \\
\text { Group }\end{array}$ & $-\mathbf{0 . 4 5 ( - 0 . 8 4}$ to & & \\
$(\mathrm{n}=209)$ & $-\mathbf{0 . 0 7})$ & & \\
\hline
\end{tabular}

Changes in outcomes are generated from mixed-effect models adjusted for age, sex and body mass index.

Abstract FRI0522 - Table 2. Effects of vitamin D status over 24 months on change in PHQ-9

\begin{tabular}{lccc}
\hline & $\begin{array}{c}\text { Mean change, } \\
(95 \% \mathrm{Cl})\end{array}$ & $\begin{array}{c}\text { Between-group difference } \\
\text { change, mean }(95 \% \mathrm{Cl})\end{array}$ & $\begin{array}{c}\mathrm{P} \text { - } \\
\text { value }\end{array}$ \\
\hline Not maintaining sufficient & $0.40(-0.18$ to & $-\mathbf{0 . 8 3}(-\mathbf{1 . 5 6}$ to $-\mathbf{0 . 1 1})$ & $\mathbf{0 . 0 2}$ \\
vitamin $\mathrm{D}(\mathrm{n}=114)$ & $0.97)$ & & \\
Maintaining sufficient vitamin & $-\mathbf{0 . 4 4}(-0.88$ to & & \\
$\mathrm{D}(\mathrm{n}=226)$ & $-0.00)$ & & \\
\hline
\end{tabular}

Changes in outcomes are generated from mixed-effect models adjusted for age, sex and body mass index. 